\title{
Rotordynamic Analysis for a Turbo-Machine with Fluid-Solid Interaction and Rotation Effects
}

\author{
Rui Wang, ${ }^{1,2}$ Yuefang Wang, ${ }^{1,2}$ and Xinglin Guo ${ }^{1,2}$ \\ ${ }^{1}$ Department of Engineering Mechanics, Dalian University of Technology, Dalian 116024, China \\ ${ }^{2}$ Dynamics and Control, State Key Laboratory of Structural Analysis for Industrial Equipment, \\ Dalian 116024, China \\ Correspondence should be addressed to Yuefang Wang, yfwang@dlut.edu.cn
}

Received 31 August 2010; Accepted 12 April 2011

Academic Editor: Victoria Vampa

Copyright (C) 2011 Rui Wang et al. This is an open access article distributed under the Creative Commons Attribution License, which permits unrestricted use, distribution, and reproduction in any medium, provided the original work is properly cited.

\begin{abstract}
The rotordynamics and fluid dynamics of a turbo-machine considering the effect of fluid-solid interaction (FSI) are numerically investigated using finite element software ADINA. The iterative method is adopted in computation of coupled fields of displacement and fluid. What distinguishes the present study from previous ones is the use of ADINA's rotational meshes and the FSI interface that separates the rotor surface from its surrounding fluid. The rotor's center orbit and frequency response as well as the transient fluid dynamics are obtained with various axial flow speeds. By including real rotating motion of the rotor, this paper presents a better way to solve complicated rotordynamic problems of turbo-machines that are operated in FSI circumstances.
\end{abstract}

\section{Introduction}

Fluid solid interaction (FSI) analyses play very important roles in understanding rotordynamics of turbo-machines. It has been noticed that fluid and solid as parts of the rotor system influence each other and severe vibrations may be excited in some situations. Particularly, this is true for machines transferring heavy fluid, for example, pumps or other turbomachines. Historically, the FSI-induced vibration was studied empirically or experimentally. For instance, Childs $[1,2]$ carried out a series of experiments to investigate the FSI forces between the impeller and the shroud of a pump as well as the resulted axial vibrations. Marscher [3] determined the "wet" critical speeds of a multistage pump rotor. Numerical methods such as the finite element method (FEM) and coupled FEM with the boundary element method (BEM) have been widely applied to various FSI problems. Bathe et al. [46] who is the developer of the ADINA software proposed application of FEM to solve the FSI problems for compressible and incompressible fluid flow by iterative method and direct 
method. Bermúdez et al. [7] demonstrated the plausibility and effectiveness of solving the FSI problems by using FEM as well. Kwon and Jo [8] modeled two 3D beams and external flow around them and used coupled lattice Boltzmann method (LBM) and FEM to analyze the characteristics of the flow field. Czygan and von Estorff $[9,10]$ and Soares et al. [11, 12] adopted both the FEM and BEM to solve the FSI problems. They used an iterative method which coupled of finite element and boundary element for the time domain to analyze fluid-solid systems. Michelin and Llewellyn Smith [13] introduced an unsteady point vortex method for two-dimensional coupled motion of a general sharp-edged solid body and a surrounding inviscid flow. The application of these methods makes integrated FSI analysis effective and computationally plausible for complex rotordynamic problems.

In this paper, the FSI problem is investigated for a turbo-machine to obtain its rotordynamic response by using finite element software ADINA with consideration of real rotating movement of the rotor surrounded by incompressible fluid flow. The fields of solid and fluid are modeled with ADINA's rotational meshes and solved iteratively for pressure and velocity of the fluid field as well as displacement of solid rotor. The first "wet" critical speed of the rotor is identified through a signal filtrating with different axial flow velocities. The natural frequency is changed from $15.63 \mathrm{~Hz}$ to $14.65 \mathrm{~Hz}$ at different Reynolds numbers.

\section{Governing Equations}

Consider a problem domain $\Omega$ consisting of a fluid part $\Omega_{f}$ and a solid part $\Omega_{s}$. For the fluid domain $\Omega_{f}$ we assume a flow of incompressible Newtonian fluid. The basic equations governing continuity and momentum are given by

$$
\begin{gathered}
\nabla \cdot\left(\rho_{s} V\right)=0, \\
\rho_{f} \frac{D v_{i}}{D t}=\rho_{f} f_{f i}+\frac{\partial T_{j i}}{\partial x_{j}}, \quad(i=1,2,3),
\end{gathered}
$$

where Einstein's summation convention is adopted here and throughout the rest of the paper. $V=\left(v_{1}, v_{2}, v_{3}\right), v_{i}$ is the velocity vector with respect to Cartesian coordinates $x_{i}, \rho_{f}$ the fluid density, $D / D t=\partial / \partial t+(V \cdot \nabla)$ is material derivative, and $f_{f i}$ are the body forces per unit mass. The stress tensor $T_{i j}$ for incompressible Newtonian fluids can be expressed as

$$
T_{i j}=\mu_{f}\left(\frac{\partial v_{j}}{\partial x_{i}}+\frac{\partial v_{i}}{\partial x_{j}}\right)-p \delta_{i j}, \quad(i=1,2,3)
$$

where $p$ is pressure and $\mu_{f}$ is dynamic viscosity. An empirical formula is used to compute Reynolds number, as follows

$$
\operatorname{Re}=\frac{\rho_{f} v_{z} D_{e}}{\mu_{f}}
$$


where $D_{e}$ is characteristic diameter denoted as

$$
D_{e}=\frac{4 A}{x}
$$

where $A$ is flow area and $\chi$ is the length of fluid-solid interface attached to the flow area.

In this model, the Reynolds number is approximately between $1.108 \times 10^{6}$ and $2.216 \times$ $10^{7}$, which is larger than its empirical critical value 1100 for turbulent flows. Hence, the numerical simulation of the flow field is carried out by using one with the turbulence model. The viscosity coefficient for the turbulent flow is modified in ADINA, as

$$
\mu=\mu_{0}+\mu_{t}
$$

where $\mu_{0}$ is the laminar viscosity and $\mu_{t}$ is its turbulence counterparts.

In the present paper, the $K-\omega$ model for high-Reynolds-number turbulences in incompressible flows is adopted. Based on the normal $K-\varepsilon$ model, a parameter $\omega$ is introduced which is related to $K$ and $\varepsilon$ as

$$
\omega \sim \frac{\varepsilon}{K}
$$

where $\varepsilon$ and $K$ are dissipation rate and kinetic energy of turbulence, respectively. Then, $\mu_{t}$ is computed by

$$
\mu_{t}=\alpha \rho_{f} \frac{K}{\omega}
$$

The governing equations for $K$ and $\omega$ are referred to ADINA [14].

The momentum equation for the solid domain $\Omega_{s}$ is expressed as

$$
\rho_{s} \frac{\partial^{2} u_{i}}{\partial t^{2}}-\frac{\partial \sigma_{i j}}{\partial x_{j}}=\rho_{s} f_{s i}, \quad(i=1,2,3),
$$

where $u_{i}$ is the displacement vector, $\sigma_{i j}$ denotes the Cauchy stress tensor, $\rho_{s}$ is the density of the solid material, and $f_{s i}$ are body forces per unit mass acting on the solid. By assuming deformation is small, the strain tensor $\varepsilon_{i j}$ is related to the displacements $u_{i}$ by the kinematic relation

$$
\varepsilon_{i j}=\frac{1}{2}\left(\frac{\partial u_{i}}{\partial x_{j}}+\frac{\partial u_{j}}{\partial x_{i}}\right), \quad(i=1,2,3) .
$$

On the fluid-solid interface the nonslip boundary conditions is applied for velocities and stresses on the interface

$$
\begin{aligned}
& v_{i, s}=v_{i, f} \\
& \sigma_{i j} n_{s j}=-T_{i j} n_{s j} \quad(i=1,2,3),
\end{aligned}
$$

where subscripts $s$ and $f$ denote the solid and fluid domains, respectively. 


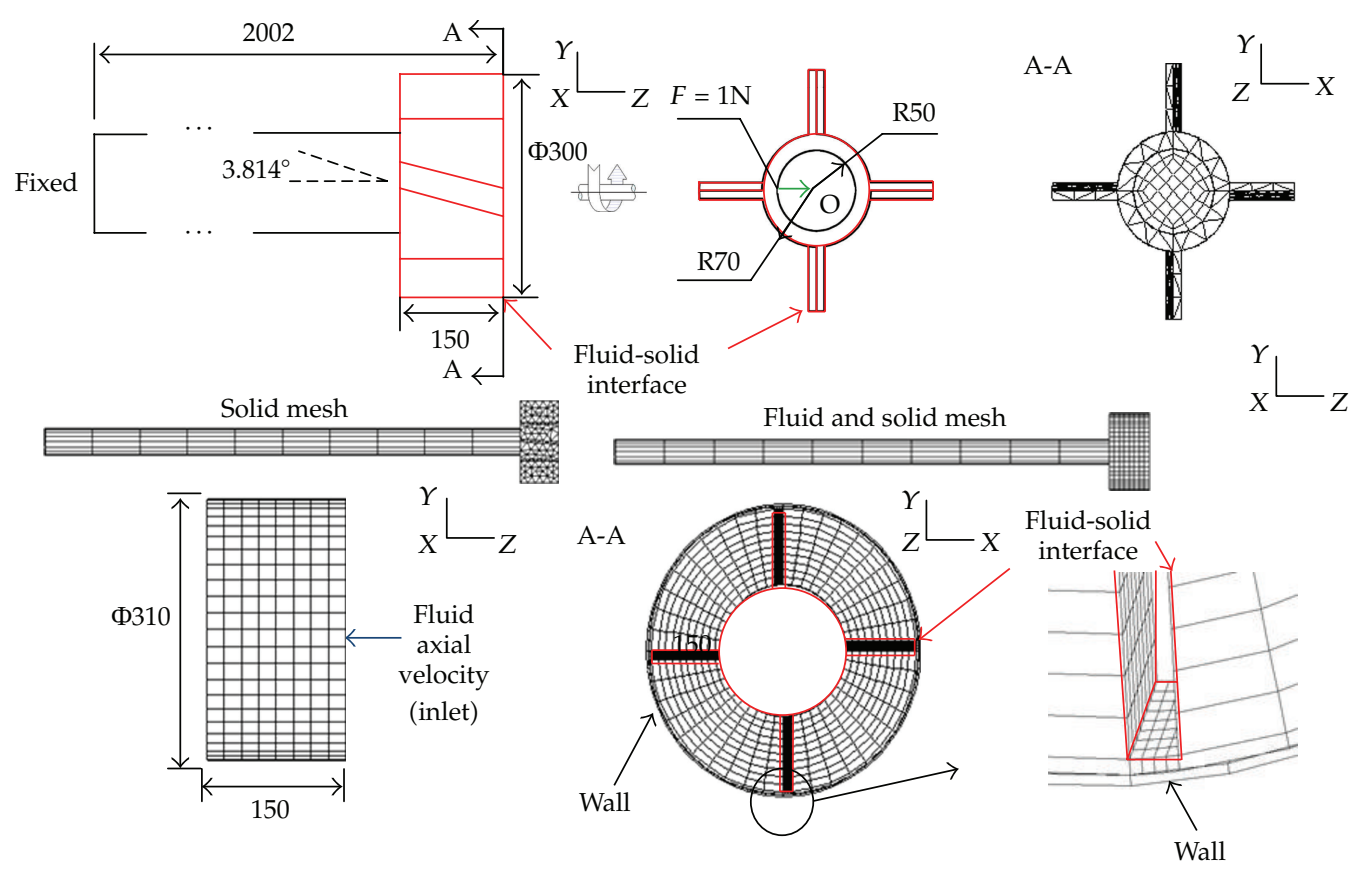

Figure 1: Computational model and finite element meshes.

Table 1: Material parameter.

\begin{tabular}{lclc}
\hline & Solid & \multicolumn{2}{c}{ Fluid } \\
\hline Young's modulus & $2.1 \times 10^{5} \mathrm{MPa}$ & Laminar viscosity & $1 \times 10^{-3} \mathrm{~Pa} \cdot \mathrm{s}$ \\
Poisson's ratio & 0.3 & & \\
Density & $7.85 \times 10^{3} \mathrm{Kg} / \mathrm{m}^{3}$ & Density & $1 \times 10^{3} \mathrm{Kg} / \mathrm{m}^{3}$ \\
\hline
\end{tabular}

\section{Computational Model and Method}

The sketch of the analysis model and its finite element meshes are shown in Figure 1 (unit: $\mathrm{mm}$ ). The radiuses of shaft and impeller are $50 \mathrm{~mm}$ and $70 \mathrm{~mm}$, respectively. The external diameter of the blade is $300 \mathrm{~mm}$. The total lengths along the $z$-axis are $2002 \mathrm{~mm}$ for the rotor and $150 \mathrm{~mm}$ for the flow field, respectively. The external diameter of the flow field is $310 \mathrm{~mm}$. The blades are straight and have a $3.814^{\circ}$ angle with respect to $z$-axis.

736 8-node-hexahedral elements and 1254 10-node-tetrahedral elements are used to mesh the solid field. For the fluid field, there are 40328 -node-hexahedral elements. In ADINA a special kind of element called rotational meshes are used to simulate the rotation of the rotor surrounded with fluid. The material parameters are displayed in Table 1.

The rotation speed of the rotor is $600 \mathrm{rpm}$. The unbalanced mass eccentricity of the rotor is $2.33 \mu \mathrm{m}$ which is applied to the model by loading it with a following force $F$ (see Figure 1 ) at the center point $O$. The magnitude of $F$ is a constant $1 \mathrm{~N}$, and its initial direction is chosen to be along the $x$ axis in the Cartesian coordinate. To simulate the rotor in real rotating status, the direction of $F$ is made to revolve synchronously with the rotor so that its loading relative to the rotation does not change. The displacement boundary condition is fixed end: $U_{x}=U_{y}=U_{z}=\theta_{x}=\theta_{y}=0$. 


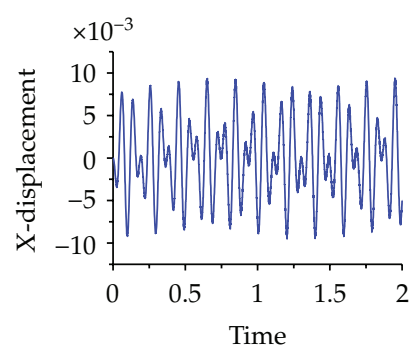

(a) $x$-displacement before filtering

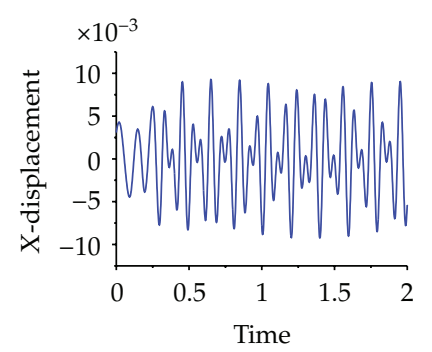

(d) $x$-displacement after filtering

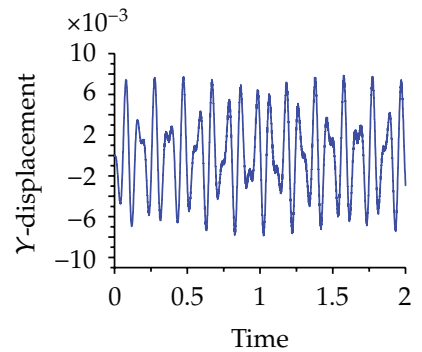

(b) $y$-displacement before filtering

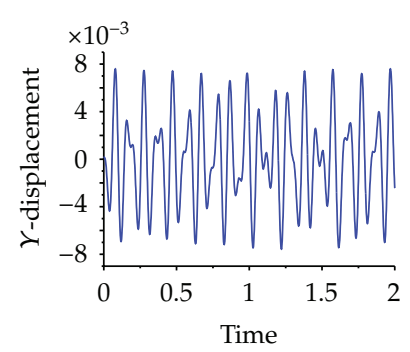

(e) $y$-displacement after filtering

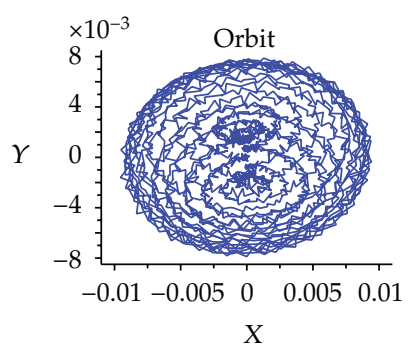

(c) Center orbit before filtering

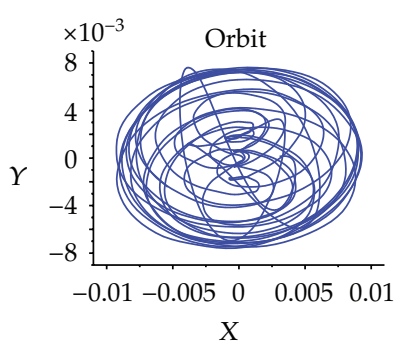

(f) Center orbit after filtering

Figure 2: Displacements and orbit of the rotor center before and after EMD filtering is performed.

The initial velocity of $v_{z}$ is negative in the $z$-direction for all fluid domains. This velocity is kept unchanged on the cross-section at the inlet. The time step of FSI is $0.001 \mathrm{~s}$ and total computation lasts $2 \mathrm{~s}$. In this case the sampling frequency for fast Fourier transform (FFT) analysis is $1000 \mathrm{~Hz}$; the Fourier length for the FFT is 1024.

The displacement $U_{x}$ and $U_{y}$ of the rotor center $\mathrm{O}$ can be obtained from the computation results and are used in signal analysis to identify frequency components. The empirical mode decomposition (EMD) method [15] is used to purify displacement signals by numerically filtrating artificial noise. Then the filtrated signals are transformed by FFT and are analyzed to determine the orbital position point $\mathrm{O}$. By cascading response of the solid part obtained from results with different flow velocities, the rotor's first critical speed upon different Reynolds numbers can be determined.

\section{Dry Critical Speed}

The rotor's first critical speed is analyzed by using the rotational meshes without considering of fluid. The results give the "dry" critical speed of the rotor that can be compared to the "wet" critical speed obtained with coupling fluid-solid interactions.

The displacements and the orbit of the axial center point $O$ are illustrated in Figure 2. The displacement results of the rotor center are filtered using EMD to remove noise. Figures 2(a), 2(b) and, 2(c) are $x$ - and $y$-directional displacements and the orbit of point $O$ before the EMD filtering, respectively. In comparison, Figures 2(d), 2(e), and 2(f) are those responses after the filtering. It is clearly seen that indeed the EMD effectively removes noise in the displacement signals while retain their dominating part in frequency domain.

Following the above filtering, the FFT analysis is carried out to identify the frequency components of the rotor response in Figure 3, where the "dry" natural frequencies are marked with their values over each peak. 


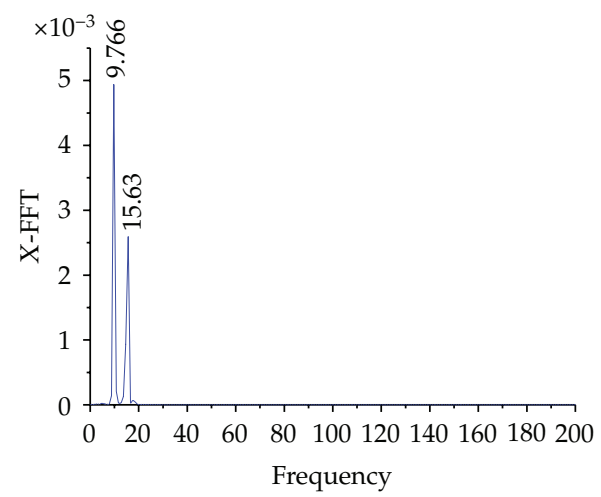

(a) $x$-directional FFT

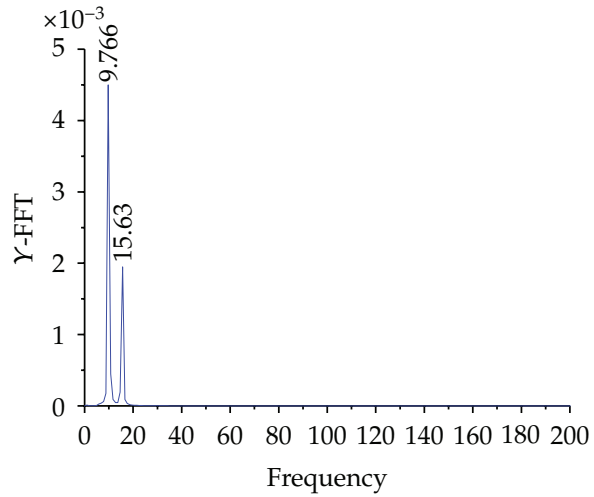

(b) $y$-directional FFT

Figure 3: FFT analysis and "dry" natural frequencies.

\section{Results of FSI Simulation}

Twelve different axial velocities at the inlet for the fluid, that is, $1 \mathrm{~m} / \mathrm{s}, 3 \mathrm{~m} / \mathrm{s}, 4 \mathrm{~m} / \mathrm{s}, 4.5 \mathrm{~m} / \mathrm{s}$, $4.85 \mathrm{~m} / \mathrm{s}, 4.9 \mathrm{~m} / \mathrm{s}, 4.95 \mathrm{~m} / \mathrm{s}, 5 \mathrm{~m} / \mathrm{s}, 6 \mathrm{~m} / \mathrm{s}, 10 \mathrm{~m} / \mathrm{s}, 15 \mathrm{~m} / \mathrm{s}$, and $20 \mathrm{~m} / \mathrm{s}$ are used to investigate the "wet" critical speed considering the fluid-solid-interaction effect. First the results with $v_{z}=5 \mathrm{~m} / \mathrm{s}$ are presented. The von Mises stress of the rotor is shown in Figure 4(a). The contour of nodal pressure of fluid is shown in Figure 4(b). The pressure of the outlet is zero which is also the reference value in computation when the boundary condition of outlet is free. Thus, pressure values of nodal points are the ones biased from the reference pressure. The maximum pressure is found on top of the pressurized surface of blades, and the minimum one is on the root of suction side. The vectors of the velocity magnitude are shown in Figure 4(c). The maximum value of velocity in the flow field is $11.337 \mathrm{~m} / \mathrm{s}$ which is found in the case of $v_{z}=5 \mathrm{~m} / \mathrm{s}$.

Eight points are picked to present the pressure distribution in Figure 5(a). Points 7561, 8551,8740 , and 9730 are on the inlet and the others are on the outlet. Points $8732,8740,9722$, and 9730 are on the pressurized side of blades while the rest are on the suction side. The pressure of points on the outlet is zero. It can be seen that pressures on the inlet are fluctuant with time and can be regarded as periodic. In addition, the pressures of points 7561 and 8740 are positive and are negative for points 8551 and 9730. The pressures of these eight points in the time domain are also shown in Figure 5(b).

Based on Figure 6 there is a short period of transient response when the rotor starts rotating along with a simultaneous axial flow (see Figures 6(a), 6(b), and 6(c)). Once the flow becomes stable, the transient response of the rotor is gone and the motion becomes periodic. Figures $6(\mathrm{~d})$ and $6(\mathrm{e})$ present the FFT results for $x$ - and $y$-displacements. There are clear peaks on FFTs in these two figures, suggesting that the first "wet" critical speed is $14.65 \mathrm{~Hz}$ when $v_{z}=5 \mathrm{~m} / \mathrm{s}$.

Next, twelve different axial velocities are used to demonstrate the "wet" critical speed. The results are listed in Table 2 based on FFT analysis for $x$ - and $y$-displacements.

As it can be seen from Table 2, the first "wet" critical speed bifurcates at $v_{z}=4.9 \mathrm{~m} / \mathrm{s}$. For $y$-displacement it drops from $15.63 \mathrm{~Hz}$ to $14.65 \mathrm{~Hz}$; the same thing happens for $x$ displacement at a slightly higher velocity $v_{z}=4.95 \mathrm{~m} / \mathrm{s}$. The bifurcation diagram of the 


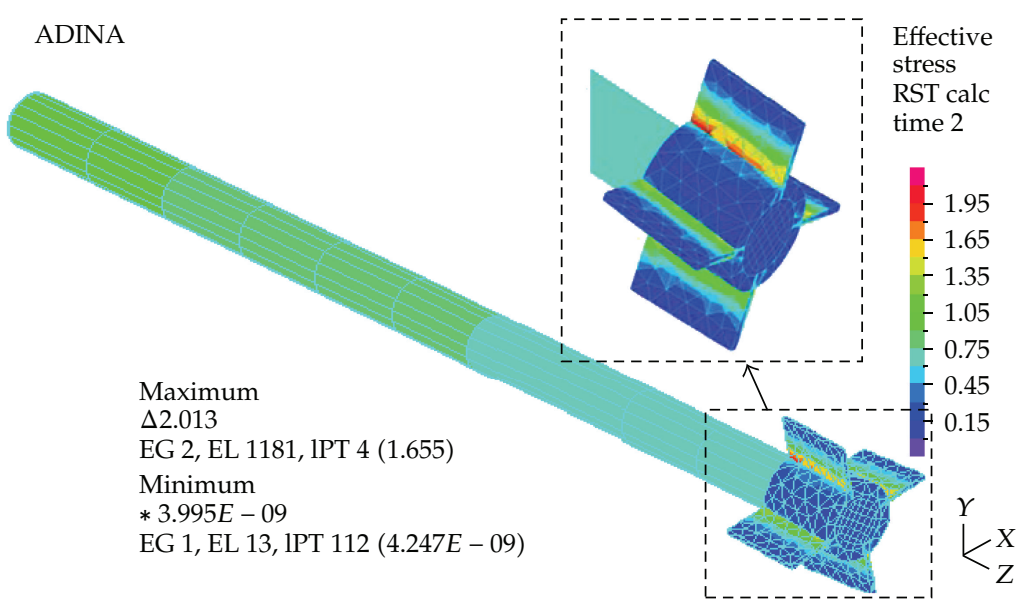

(a) Von Mises stress distribution

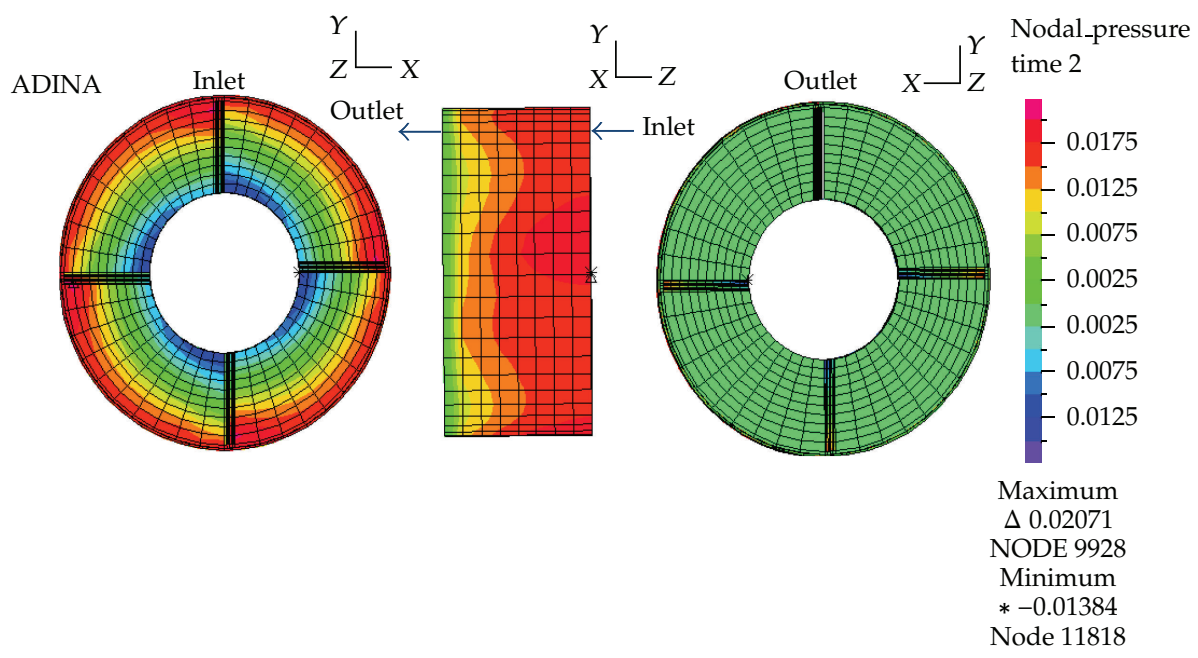

(b) Nodal pressure

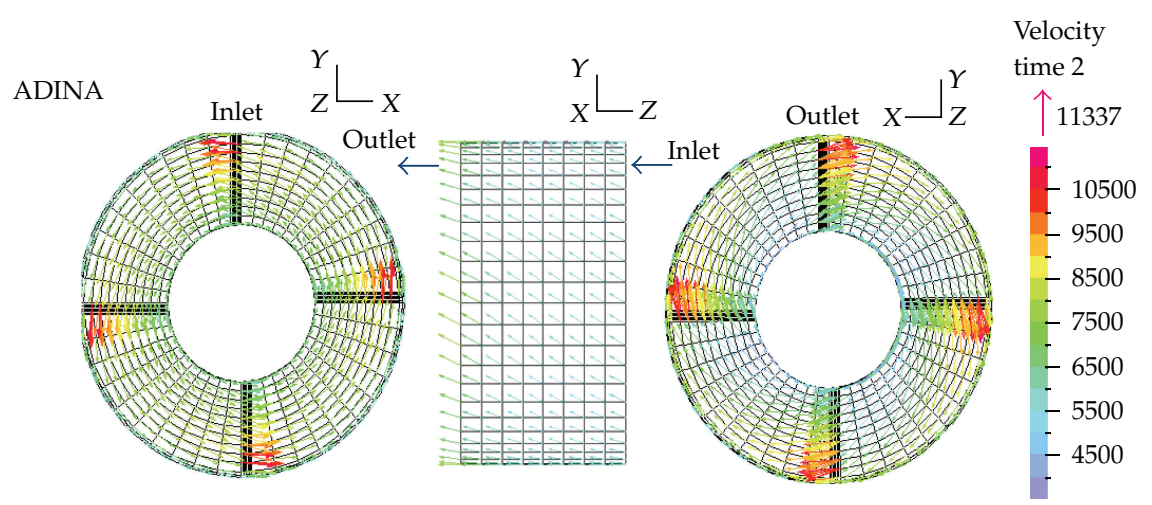

(c) Velocity

Figure 4: The results of FSI. 


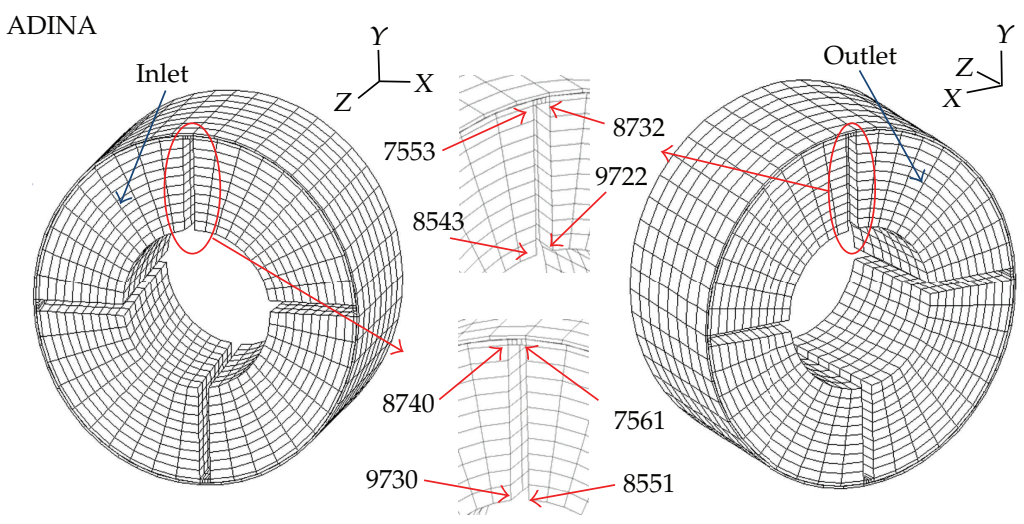

(a) Nodal positions of the picked points

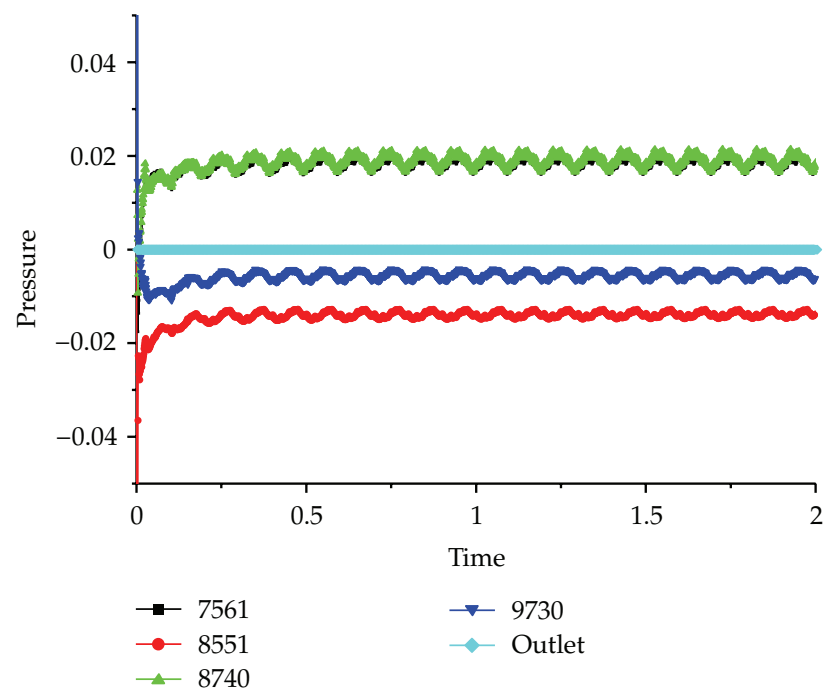

(b) Nodal pressure

Figure 5: Pressure values for points on the inlet and outlet.

Table 2: The results of first critical speed with various axial velocities.

\begin{tabular}{lccccccccccccc}
\hline Velocity & $(\mathrm{m} / \mathrm{s})$ & 1 & 3 & 4 & 4.5 & 4.85 & 4.9 & 4.95 & 5 & 6 & 10 & 15 & 20 \\
\hline $\begin{array}{l}\text { First } \\
\text { critical } \\
\text { speed }\end{array}$ & X-FFT & 15.63 & 15.63 & 15.63 & 15.63 & 15.63 & 15.63 & 14.65 & 14.65 & 14.65 & 14.65 & N/A & N/A \\
\hline
\end{tabular}

FFTs with axial velocity is shown in Figure 7. The FFT results of $x$-displacements when $v_{z}=$ $10 \mathrm{~m} / \mathrm{s}, v_{z}=15 \mathrm{~m} / \mathrm{s}$, and $v_{z}=20 \mathrm{~m} / \mathrm{s}$ are shown in Figure 8 .

Based on the above results, the rotor's first critical speed drops and bifurcates with an increasing fluid axial velocity. The peaks that represent critical speeds on the FFT results become unclear with faster flow and almost disappear when $v_{z}=15 \mathrm{~m} / \mathrm{s}$. The peaks eventually cannot be identified at all when $v_{z}=20 \mathrm{~m} / \mathrm{s}$. 


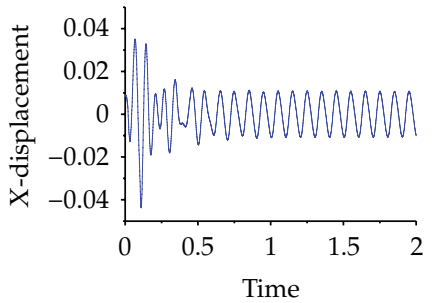

(a) $x$-displacement versus time

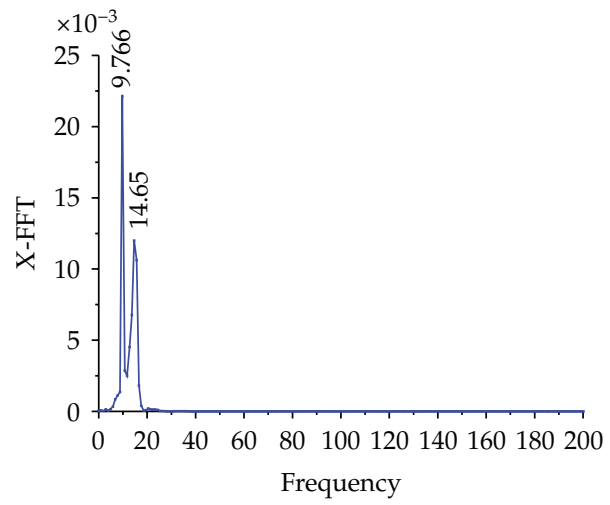

(d) $x$-directional FFT

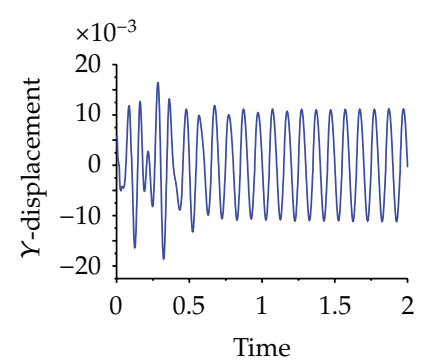

(b) $y$-displacement versus time

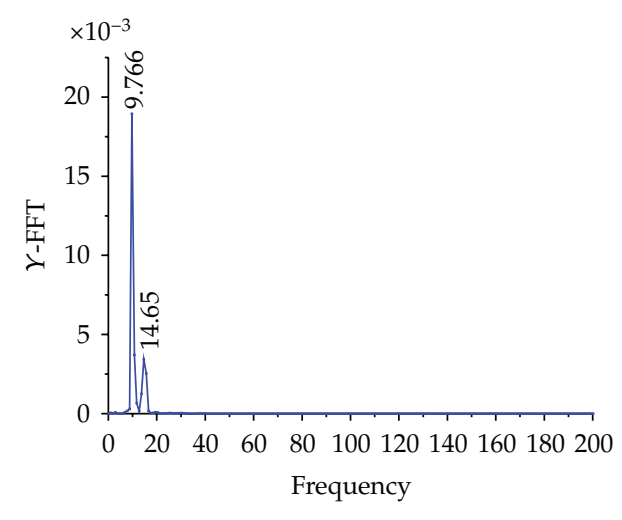

(e) $y$-directional FFT

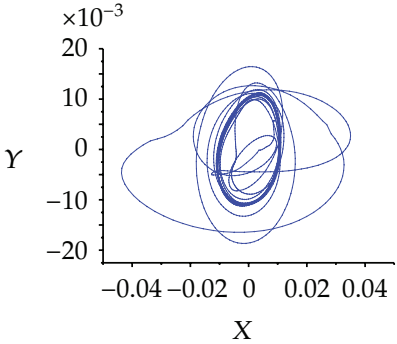

(c) Center orbit

Figure 6: Results of FSI when $v_{z}=5 \mathrm{~m} / \mathrm{s}$.

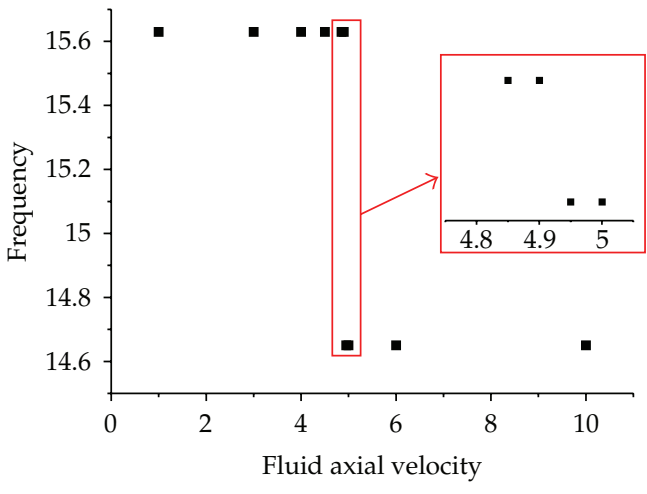

- X-FFT

(a) Frequency of $x$-displacement versus flow speed 


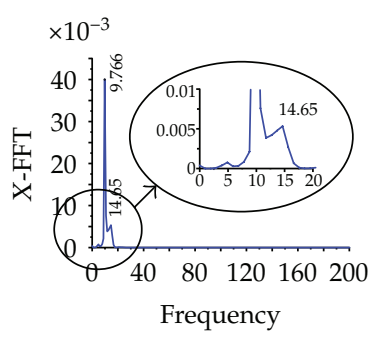

(a) $v_{z}=10 \mathrm{~m} / \mathrm{s}$

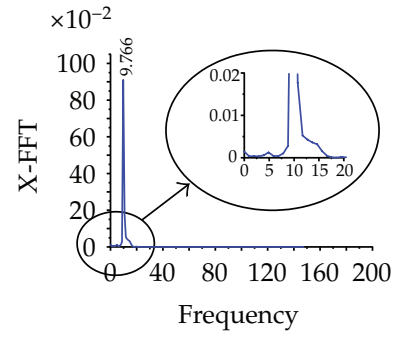

(b) $v_{z}=15 \mathrm{~m} / \mathrm{s}$

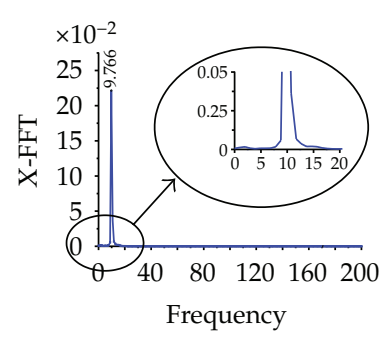

(c) $v_{z}=20 \mathrm{~m} / \mathrm{s}$

Figure 8: The FFT results of $x$-displacement when $v_{z}=10 \mathrm{~m} / \mathrm{s}, v_{z}=15 \mathrm{~m} / \mathrm{s}$, and $v_{z}=20 \mathrm{~m} / \mathrm{s}$.

speeds are obtained with several different axial velocities of fluid flow. The results of FEM with fluid-solid interaction can be determined and processed by the FFT analysis with signals purified. The orbit of the center point in time domain is obtained by the EMD filter. Then, the "wet" first critical speed can be determined.

As the increase of the fluid axial velocity, the "wet" first critical speed changes from $15.63 \mathrm{~Hz}$ to $14.65 \mathrm{~Hz}$. Then, the peak gets lower and cannot be distinguished at higher Reynolds numbers. The present study shows that the first critical speed changes with the velocities of the flow. It means the structure has different "wet" first critical speeds in different Reynolds numbers with various flow conditions.

\section{Acknowledgment}

The authors are grateful for supports from the Exploration Projects on Nuclear Pumps of Dalian University of Technology, the National Science Foundation of China (10721062), and the State Key Development Program for Basic Research of China (Projects 2009CB724300, 2011CB706504).

\section{References}

[1] D. W. Childs, "Fluid-structure interaction forces at pump-impeller-shroud surfaces for rotordynamic calculations," Journal of Vibration and Acoustics, vol. 111, no. 7, pp. 216-225, 1989.

[2] D. W. Childs, "Fluid-structure interaction forces at pump-impeller-shroud surfaces for axial vibration analysis," Journal of Vibration and Acoustics, vol. 113, no. 1, pp. 108-115, 1991.

[3] W. D. Marscher, "Analysis and test of multistage pump 'wet' critical speeds," Tribology Transactions, vol. 34, no. 3, pp. 445-457, 1991.

[4] K. J. Bathe, H. Zhang, and M. H. Wang, "Finite element analysis of incompressible and compressible fluid flows with free surfaces and structural interactions," Computers and Structures, vol. 56, no. 2-3, pp. 193-213, 1995.

[5] K. J. Bathe, H. Zhang, and S. Ji, "Finite element analysis of fluid flows fully coupled with structural interactions," Computers and Structures, vol. 72, no. 1-3, pp. 1-16, 1999.

[6] K. J. Bathe and H. Zhang, "Finite element developments for general fluid flows with structural interactions," International Journal for Numerical Methods in Engineering, vol. 60, no. 1, pp. 213-232, 2004.

[7] A. Bermúdez, R. Durán, and R. Rodríguez, "Finite element analysis of compressible and incompressible fluid-solid systems," Mathematics of Computation, vol. 67, no. 221, pp. 111-136, 1998.

[8] Y. W. Kwon and J. C. Jo, “3D modeling of fluid-structure interaction with external flow using coupled LBM and FEM," Journal of Pressure Vessel Technology, vol. 130, no. 2, pp. 021301-1-021301-8, 2008.

[9] O. Czygan and O. von Estorff, "FEM/BEM coupling for fluid-structure interaction including nonlinear effects," in Proceedings of the BEM 22 Conference, vol. 8, pp. 501-509, Cambridge, UK, 2000. 
[10] O. Czygan and O. von Estorff, "Fluid-structure interaction by coupling BEM and nonlinear FEM," Engineering Analysis with Boundary Elements, vol. 26, no. 9, pp. 773-779, 2002.

[11] D. Soares Jr., O. von Estorff, and W. J. Mansur, "Iterative coupling of BEM and FEM for nonlinear dynamic analyses," Computational Mechanics, vol. 34, no. 1, pp. 67-73, 2004.

[12] D. Soares Jr., O. von Estorff, and W. J. Mansur, “Efficient non-linear solid-fluid interaction analysis by an iterative BEM/FEM coupling," International Journal for Numerical Methods in Engineering, vol. 64, no. 11, pp. 1416-1431, 2005.

[13] S. Michelin and S. G. Llewellyn Smith, "An unsteady point vortex method for coupled fluid-solid problems," Theoretical and Computational Fluid Dynamics, vol. 23, no. 2, pp. 127-153, 2009.

[14] ADINA R\&D, Theory and Modeling Guide Volume III: ADINA CFD \& FSI, 2006.

[15] D. Yu, J. Cheng, and Y. Yang, "Application of EMD method and Hilbert spectrum to the fault diagnosis of roller bearings," Mechanical Systems and Signal Processing, vol. 19, no. 2, pp. 259-270, 2005. 


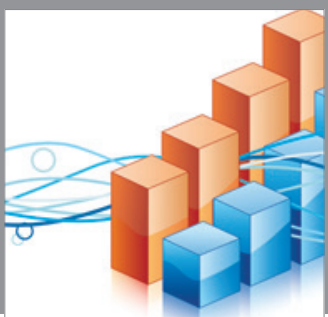

Advances in

Operations Research

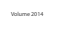

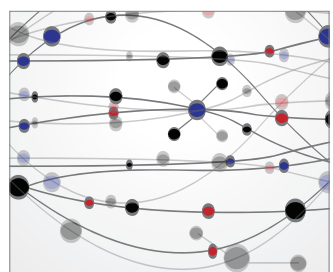

\section{The Scientific} World Journal
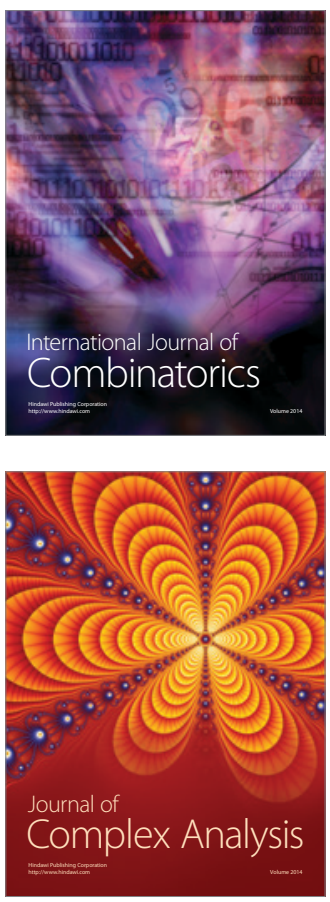

International Journal of

Mathematics and

Mathematical

Sciences
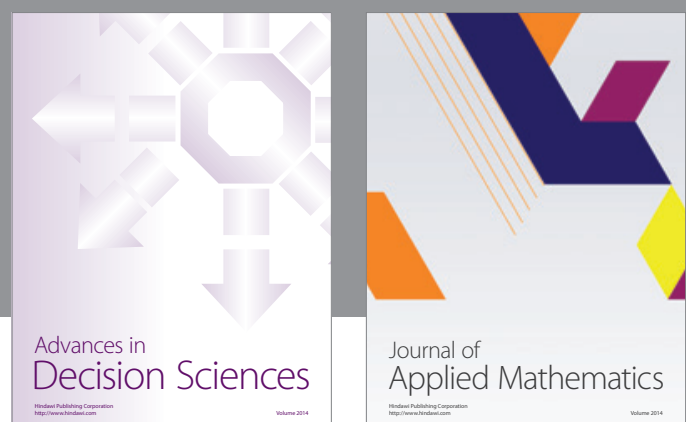

Journal of

Applied Mathematics
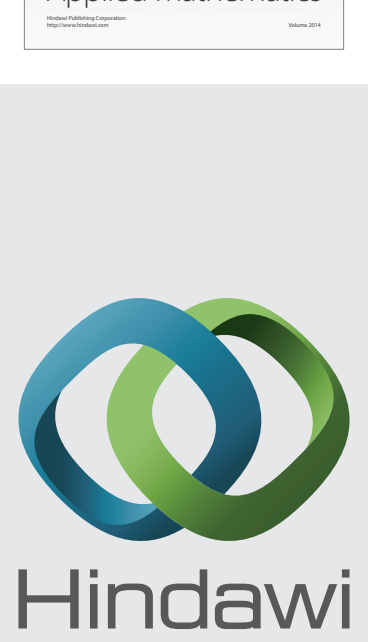

Submit your manuscripts at http://www.hindawi.com
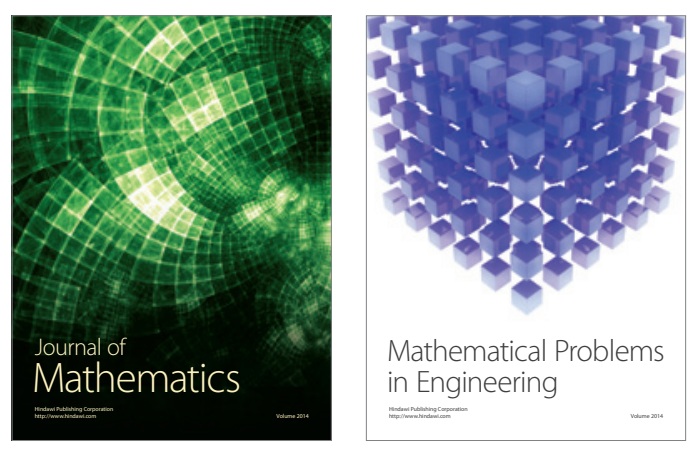

Mathematical Problems in Engineering
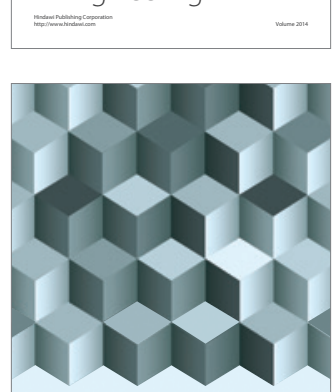

Journal of

Function Spaces
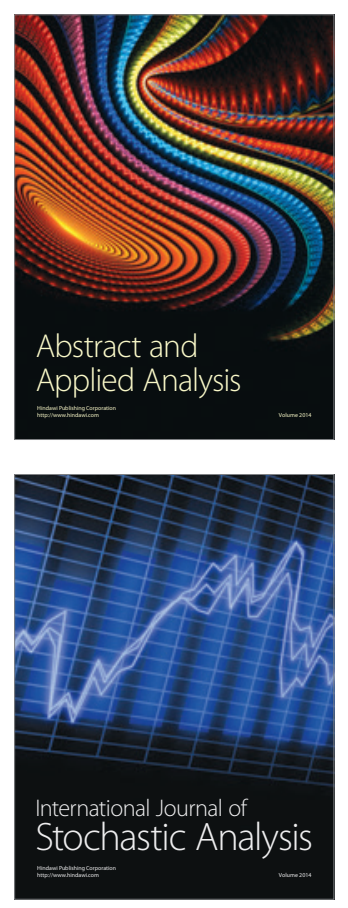

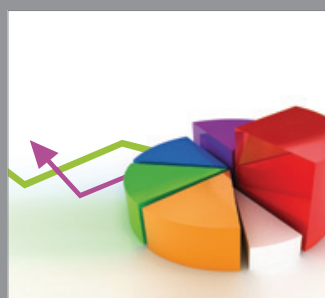

ournal of

Probability and Statistics

Promensencen
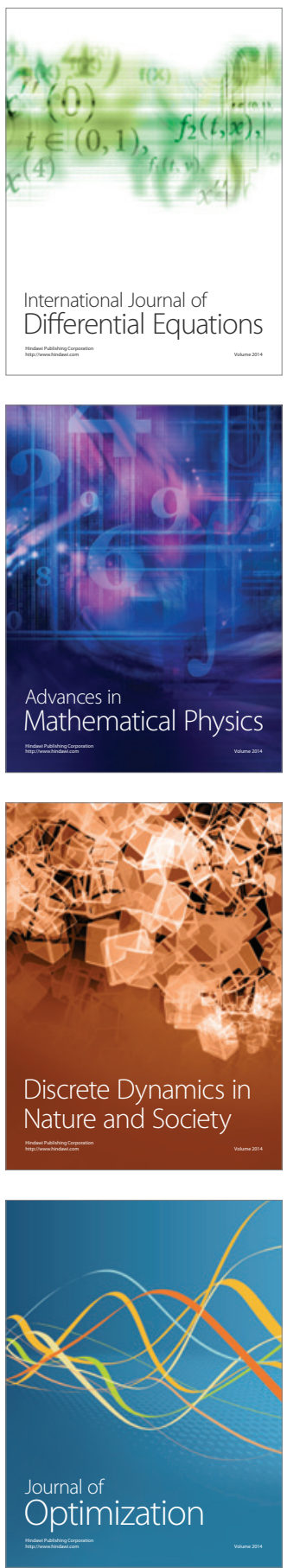